\title{
Angiospermic flora of Rajshahi district, Bangladesh
}

\author{
A. H. M. Mahbubur Rahman \\ Department of Botany, University of Rajshahi, Rajshahi-6205, Bangladesh
}

E-mail address:

ahmmahbubur_rahman@yahoo.com

\section{To cite this article:}

A. H. M. Mahbubur Rahman. Angiospermic Flora of Rajshahi District, Bangladesh. American Journal of Life Sciences. Vol. 1, No. 3, 2013, PP. 105-112. doi: 10.11648/j.ajls.20130103.15

\begin{abstract}
The present article focuses on an inventory of angiospermic flora of Rajshahi district. Using traditional techniques data have been collected from the study area during July 2010 to June 2012. In this preliminary assessment, a total of 425 angiosperm species under 321 genera and 108 families were recorded. Habit analysis shows that herbs, shrubs, climbers and trees are represented by 186, 74, 52 and 113 species, respectively. Out of the total number of species 281 were commom, 76 were very common, 57 were vulnerable and 11 were endangered species in the study area.
\end{abstract}

Keywords: Preliminary Assessment, Angiospermic Flora, Rajshahi District, Bangladesh

\section{Introduction}

Rajshahi district is a district in north-western Bangladesh. It is a part of the Rajshahi division. The metropolitan city of Rajshahi is in Rajshahi district. The Rajshahi district is bounded by Naogaon district to the north, Natore district to the east, and Chapai Nawabganj district and the river Padma to the south. The Rajshahi district has a sub-tropical monsoon climate, typical of Bangladesh, which falls within a low rainfall zone of the country. 75 percent rainfall occurs during June-September. The annual rainfall is $1350 \mathrm{~mm}$. Temperature of the area is low in January varies from $9.0^{\circ} \mathrm{C}$ to $14.1^{\circ} \mathrm{C}$. From February an increasing trend of temperature is found up to April and thereafter temperature start to decline. In April temperature varies from $22.6^{\circ} \mathrm{C}$ to $36.9^{\circ} \mathrm{C}$. The mean relative humidity is found to be low in March (65\%) and high in July-September (88-89\%) [2].

The plants of Rajshahi district exhibit diverse habitats, such as wetland, scrub jungles, fallow lands etc. which support luxuriant formation of angiosperms and play a vital role in the local economy, environment and primary healthcare system. The importance of studying local floristic diversity has been realized and carried out in Bangladesh by Khan et al. [6], Rahman and Hassan [12], Uddin and Rahman [20], Khan and Huq [7], Uddin and Hassan [21]. Recently Tutul et al. [18, 19] and Rahman et al. [13, 14] conducted the inventorying of angiosperm diversity of Dhamrai Upazilla of Dhaka and Runctia Sal forest of Bangladesh. The present study will be made an inventory of the angiosperm plant species of Rajshahi district, Bangladesh.

\section{Materials and Methods}

Angiospermic plant species growing throughout in the Rajshahi district of Bangladesh was carried out from July 2010 to June 2012. A total of 425 species under 321 genera and 108 families were collected and identified. A survey on the determination of the location of different species was made and a list was prepared to be acquainted with the plants available in the selected area. All the species were noted and time to time the areas were visited to see when they flowered. For the morphological study, different types of species were examined again and again in order to see if there was any variation or not. They were collected at flowering stages and herbarium specimens were prepared as vouchers. In this practice standard method was followed. In this regard different types of plant species were collected from different habitats. All the collected plant specimens were kept in the Herbarium, Department of Botany, and University of Rajshahi, Bangladesh.

The collected specimens were identified studying related taxonomic books and booklets from the library of Rajshahi University. The major collected materials were identified and described up to species with the help of [3], [4], [8] and [10]. In some cases [5], [1], [15-17] and [11] were consulted. For the current name and up to date nomenclature [5], [1] and [9] were also consulted. 


\section{Results and Discussion}

Based on this study, a preliminary list of angiospermic flora of the Rajshahi district was made that includes 425 angiosperm species under 321 genera and 108 families. For each species botanical name, local name, habit, plant population and family were provided. Of 425 species recorded here, herbs are represented by 186 , trees by 113 , shrubs by 74, and climber by 52 species. Out of the total number of species 281 were common, 76 were very common, 57 were vulnerable and 11 were endangered species (Table 1).

Distribution of angiosperm species in the families shows variation. The family Asteraceae is represented by 35 species and each of Caesalpiniaceae and Solanaceae is represented by 15 species. Cucurbitaceae is represented by 23 species, Mimosaceae by 10 species, Moraceae by 9 species, Poaceae by 14 species, Rutaceae by 11 species, Fabaceae by 26 species and Amaranthaceae by 9 species. A single species in each was recorded by 52 families while two to seven species in each was recorded by 46 families (Table 1).

Among the species studied, Acacia mangium Willd., Blumea laciniata (Roxb.) DC., Couroupita guianensis Aubl., Cresentia cujette L., Jacaranda mimosifolia D. Don., Tagetes patula L., Tabebuia rosea (Bertol.) DC., Wedelia trilobata (L.) A. S. Hitchc. and Xylia dolabiformis Benth. Have been reported here for the first time from Rajshahi district, and Parthenium hysterophorus Linn. Was a new record from Bangladesh.

Table 1. Angiospermic flora in the Rajshahi district of Bangladesh

\begin{tabular}{|c|c|c|c|c|c|}
\hline $\mathbf{S} / \mathbf{N}$ & Botanical name & Local name & Family name & Habit & Plant population \\
\hline 1 & Abelmoshus esculentus (L.) Moen. & Bhindi & Malvaceae & Herb & $\mathrm{VC}$ \\
\hline 2 & Abroma augusta ( L.) L. f. & Ulatkambal & Sterculiaceae & Shrub & VS \\
\hline 3 & Abrus precatorius L. & Kuch & Fabaceae & Climber & ES \\
\hline 4 & Abutilon indicum (L.) Sweet & Potari & Malvaceae & Herb & $\mathrm{C}$ \\
\hline 5 & Acacia auriculiformis Benth. & Akasmoni & Mimosaceae & Tree & $\mathrm{VC}$ \\
\hline 6 & Acacia mangium Willd. & Mangium & Mimosaceae & Tree & $\mathrm{C}$ \\
\hline 7 & Acacia catechu (L. f.) Willd. & Khair & Mimosaceae & Tree & $\mathrm{C}$ \\
\hline 8 & Acacia nilotica (L.) Del. & Babla & Mimosaceae & Tree & $\mathrm{C}$ \\
\hline 9 & Acalypha indica $\mathrm{L}$. & Muktajhuri & Euphorbiaceae & Herb & $\mathrm{VC}$ \\
\hline 10 & Achyranthes aspera L. & Apang & Amaranthaceae & Herb & $\mathrm{VC}$ \\
\hline 11 & Acorus calamus $\mathrm{L}$. & Bach & Araceae & Herb & $\mathrm{C}$ \\
\hline 12 & Adhatoda vasica Nees & Basak & Acanthaceae & Shrub & $\mathrm{C}$ \\
\hline 13 & Aegle marmelos (L.) Correa & Bel & Rutaceae & Tree & $\mathrm{C}$ \\
\hline 14 & Aerva sanguinolenta (L.) Bl. & Nuria & Amaranthaceae & Herb & $\mathrm{C}$ \\
\hline 15 & Agave americana $\mathrm{L}$. & Agave & Agavaceae & Herb & $\mathrm{C}$ \\
\hline 16 & Ageratum conyzoides (L.) L. & Ochunti & Asteraceae & Herb & $\mathrm{VC}$ \\
\hline 17 & Albizia lebbek (L.) Benth & Sirish & Mimosaceae & Tree & $\mathrm{C}$ \\
\hline 18 & Albizia lucida Benth. & Silkoroi & Mimosaceae & Tree & $\mathrm{C}$ \\
\hline 19 & Albizia procera Benth. & Koroi & Mimosaceae & Tree & $\mathrm{C}$ \\
\hline 20 & Albizia richardiana King \& Prain & Gagansirish & Mimosaceae & Tree & \\
\hline 21 & Allium cepa $\mathrm{L}$. & Piaj & Liliaceae & Herb & $\mathrm{C}$ \\
\hline 22 & Allium sativum $\mathrm{L}$. & Rosun & Liliaceae & Herb & $\mathrm{C}$ \\
\hline 23 & Alocasia indica (Roxb.) Schott. & Mankachu & Araceae & Herb & $\mathrm{C}$ \\
\hline 24 & Aloe vera $(\mathrm{L})$ Burm. f.. & Gritakumari & Aloeaceae & Herb & $\mathrm{C}$ \\
\hline 25 & Alstonia scholaris (L.) R. Br. & Chatim & Apocynaceae & Tree & $\mathrm{C}$ \\
\hline 26 & Alternanthera philoxeroides (Mart.) Griseb. & Helencha & Amaranthaceae & Herb & $\mathrm{C}$ \\
\hline 27 & Alternanthera sessilis R.Br. & Chanchi & Amaranthaceae & Herb & $\mathrm{VC}$ \\
\hline 28 & Amaranthus lividus Roxb. & Gobranotey & Amaranthaceae & Herb & $\mathrm{VC}$ \\
\hline 29 & Amaranthus spinosus L. & Kantanotey & Amaranthaceae & Herb & $\mathrm{VC}$ \\
\hline 30 & Amaranthus tricolor L. & Lalshak & Amaranthaceae & Herb & $\mathrm{VC}$ \\
\hline 31 & Amaranthus viridis $\mathrm{L}$. & Noteyshak & Amaranthaceae & Herb & $\mathrm{VC}$ \\
\hline 32 & Ammania baccifera $\mathrm{L}$. & Janglimehendi & Lythraceae & Herb & $\mathrm{C}$ \\
\hline 33 & Amorphophallus campanulatus (Roxb) Bl. Ex Dence & Olkochu & Araceae & Herb & $\mathrm{C}$ \\
\hline 34 & Annanas sativus Schult. f. & Anaras & Bromeliaceae & Herb & VS \\
\hline 35 & Anagallis arvensis L. & Pimpernel & Primulaceae & Herb & $\mathrm{C}$ \\
\hline 36 & Anacardium occidentale L. & Kajubadam & Anacardiaceae & Tree & VS \\
\hline 37 & Andrographis paniculata Wall ex Nees & Kalomegh & Acanthaceae & Herb & $\mathrm{C}$ \\
\hline 38 & Anisomeles indica (L.) Kuntze. & Gobura & Lamiaceae & Herb & $\mathrm{C}$ \\
\hline 39 & Annona reticulata $\mathrm{L}$. & Nona & Annonaceae & Tree & $\mathrm{C}$ \\
\hline 40 & Annona squamosa $\mathrm{L}$. & Ata & Annonaceae & Tree & $\mathrm{C}$ \\
\hline 41 & Anthocephalus chinensis (Lamk.) Rich. ex Walp. & Kadam & Rubiaceae & Tree & $\mathrm{C}$ \\
\hline 42 & Antirrhinum majus L. & Snapdragon & Scrophulariaceae & Herb & VS \\
\hline 43 & Aphanamixis polystachya (Wall.) R N Park. & Pitraj & Meliaceae & Tree & VS \\
\hline 44 & Aquilaria agallocha Roxb. & Agar & Thymelaeaceae & Tree & VS \\
\hline 45 & Arachis hypogea $\mathrm{L}$. & Chenabadam & Fabaceae & Herb & $\mathrm{C}$ \\
\hline 46 & Areca catechu L. & Supari & Arecaceae & Tree & $\mathrm{VC}$ \\
\hline 47 & Argemone maxicana $\mathrm{L}$. & Shialkanta & Papaveraceae & Herb & $\mathrm{C}$ \\
\hline 48 & Aristolachia indica $\mathrm{L}$. & Iswarmul & Aristolachiaceae & Climber & VS \\
\hline 49 & Artocarpus heterophyllus Lamk. & Kathal & Moraceae & Tree & $\mathrm{VC}$ \\
\hline 50 & Artocarpus lacucha Buch.-Ham. & Deua & Moraceae & Tree & VS \\
\hline
\end{tabular}




\begin{tabular}{|c|c|c|c|c|c|}
\hline 51 & Asparagus racemosus $\mathrm{L}$. & Satamuli & Liliaceae & Climber & $\mathrm{C}$ \\
\hline 52 & Averrhoa carambola $\mathrm{L}$. & Kamranga & Oxalidaceae & Tree & $\mathrm{R}$ \\
\hline 53 & Averrhoa bilimbi L. & Kamranga & Oxalidaceae & Tree & VS \\
\hline 54 & Azadirachta indica A. Juss. & Neem & Meliaceae & Tree & $\mathrm{VC}$ \\
\hline 55 & Baccaurea ramiflora Lour. & Lotkan & Euphorbiaceae & Tree & $\mathrm{C}$ \\
\hline 56 & Bacopa monnieri (L.) Pennell & Brammishak & Scrophulariaceae & Herb & VS \\
\hline 57 & Bambusa balcooa Roxb. & Valkabans & Poaceae & Tree & $\mathrm{C}$ \\
\hline 58 & Bambusa tulda Roxb. & Tollabans & Poaceae & Tree & $\mathrm{C}$ \\
\hline 59 & Barringtonia acutangula (L.) Gaertn & Hijol & Lecythidaceae & Tree & ES \\
\hline 60 & Barleria cristata $\mathrm{L}$. & Swetjanti & Acanthaceae & Herb & $\mathrm{C}$ \\
\hline 61 & Basella alba $\mathrm{L}$. & Puishak. & Basellaceea & Climber & $\mathrm{C}$ \\
\hline 62 & Bauhinia acuminata $\mathrm{L}$. & Kanchan & Caesalpiniaceae & Tree & $\mathrm{C}$ \\
\hline 63 & Benincasa hispida (Thunb.) Cogn. & Chalkumra & Cucurbitaceae & Climber & $\mathrm{C}$ \\
\hline 64 & Beta vulgaris $\mathrm{L}$. & Beetpalang & Chenopodiaceae & Herb & VS \\
\hline 65 & Biophytum sensitivum (L.) DC. & Panilajuk & Oxalidaceae & Herb & VS \\
\hline 66 & Bixa orellana L. & Jafran, Sidur & Bixaceae & Tree & ES \\
\hline 67 & Blumea lacera (Burm.f.) DC. & Kukurshunga & Asteraceae & Herb & $\mathrm{C}$ \\
\hline 68 & Blumea laciniata DC. & Kukurshunga & Asteraceae & Herb & $\mathrm{C}$ \\
\hline 69 & Boerhaavia repens $\mathrm{L}$. & Punarnava & Nyctaginaceae & Climber & $\mathrm{C}$ \\
\hline 70 & Bombax ceiba L. & Simul & Bombacaceae & Tree & $\mathrm{C}$ \\
\hline 71 & Bombax insigne Wall. & Varotiosimul & Bombacaceae & Tree & ES \\
\hline 72 & Borassus flabellifer $\mathrm{L}$. & Tal & Arecaceae & Tree & $\mathrm{C}$ \\
\hline 73 & Bouganvillea spectabilis Willd. & Baganbilas & Nyctaginaceae & Climber & $\mathrm{C}$ \\
\hline 74 & Brassica napus $\mathrm{L}$. & Sarisha & Brassicaceae & Herb & $\mathrm{C}$ \\
\hline 75 & Brassica juncea $\mathrm{L}$. & Raisarisha & Brassicaceae & Herb & $\mathrm{C}$ \\
\hline 76 & Brassica oleracea L. var capitata & Badhakopi & Brassicaceae & Herb & $\mathrm{C}$ \\
\hline 77 & Brassica oleracea L. var botrydis & Phulkapi & Brassicaceae & Herb & $\mathrm{C}$ \\
\hline 78 & Brassica rapa $\mathrm{L}$. & Salgom & Brassicaceae & Herb & $\mathrm{C}$ \\
\hline 79 & Butea monosperma (Lam.) Taub. & Palas & Fabaceae & Tree & $\mathrm{C}$ \\
\hline 80 & Caesalpinia bonduc (L.) Roxb. & Nata & Caesalpiniaceae & Shrub & $\mathrm{C}$ \\
\hline 81 & Caesulia auxillaris Roxb. & Caesulia & Asteraceae & Herb & $\mathrm{C}$ \\
\hline 82 & Cajanus cajan (L.) Huth. & Arhar & Fabaceae & Shrub & $\mathrm{C}$ \\
\hline 83 & Calamus rotung $\mathrm{L}$. & Beet & Arecaceae & Shrub & VS \\
\hline 84 & Calendula officinalis Hook. & Calendula & Asteraceae & Herb & $\mathrm{C}$ \\
\hline 85 & Callistemon citrinus Stapf. & Bottlebrass & Myrtaceae & Tree & $\mathrm{C}$ \\
\hline 86 & Callistephus chinensis L. & Aster & Asteraceae & Herb & $\mathrm{C}$ \\
\hline 87 & Calotropis gigantea $\mathrm{Br}$. & Baraakanda & Asclepiadaceae & Shrub & $\mathrm{C}$ \\
\hline 88 & Calotropis procera $\mathrm{Br}$. & Akanda & Asclepiadaceae & Shrub & $\mathrm{C}$ \\
\hline 89 & Canna indica $\mathrm{L}$. & Kolabati & Cannaceae & Herb & $\mathrm{C}$ \\
\hline 90 & Capsicum frutescens $\mathrm{L}$. & Morich & Solanaceae & Herb & $\mathrm{C}$ \\
\hline 91 & Careya arborea Roxb. & Kumvi & Lecythidaceae & Tree & VS \\
\hline 92 & Carica papaya $\mathrm{L}$. & Pepe & Caricaceae & Tree & $\mathrm{VC}$ \\
\hline 93 & Carissa carandus $\mathrm{L}$. & Karamcha & Apocynaceae & Shrub & $\mathrm{VC}$ \\
\hline 94 & Cassia alata $\mathrm{L}$. & Dadmardan & Caeslpiniaceae & Shrub & $\mathrm{C}$ \\
\hline 95 & Cassia fistula $\mathrm{L}$. & Badarlathi & Caeslpiniaceae & Tree & $\mathrm{C}$ \\
\hline 96 & Cassia sophera L. & Kalkashundha & Caeslpiniaceae & Shrub & $\mathrm{C}$ \\
\hline 97 & Cassia obtusifolia $\mathrm{L}$. & Kalkashundha & Caeslpiniaceae & Shrub & $\mathrm{C}$ \\
\hline 98 & Cassia tora $\mathrm{L}$. & Kalkashundha & Caeslpiniaceae & Shrub & $\mathrm{C}$ \\
\hline 99 & Cassia siamea Lam. & Minjuri & Caeslpiniaceae & Tree & $\mathrm{C}$ \\
\hline 100 & Cassia renigera $\mathrm{L}$. & Minjiri & Caeslpiniaceae & Tree & $\mathrm{C}$ \\
\hline 101 & Cassia grandis $\mathrm{L}$. & Mingiri & Caeslpiniaceae & Tree & $\mathrm{C}$ \\
\hline 102 & Casuarina equisetifolia $\mathrm{L}$. & Jhau & Casuarinaceae & Tree & $\mathrm{C}$ \\
\hline 103 & Catharanthus roseus (L.) G. Don. & Nayantara & Apocynaceae & Herb & $\mathrm{VC}$ \\
\hline 104 & Celosia cristata $\mathrm{L}$. & Morogphul & Amaranthaceae & Herb & $\mathrm{C}$ \\
\hline 105 & Centella asiatica (L.) Urban. & Thankuni & Apiaceae & Herb & $\mathrm{C}$ \\
\hline 106 & Cestrum nocturnum L. & Hasnahena & Solanaceae & Shrub & $\mathrm{C}$ \\
\hline 107 & Ceratophyllum demersum $\mathrm{L}$. & Jhanjhi & Ceratophyllaceae & Herb & $\mathrm{C}$ \\
\hline 108 & Chenopodium album $\mathrm{L}$. & Botuashak & Chenopodiaceae & Herb & $\mathrm{C}$ \\
\hline 109 & Chenopodium ambrosoides $\mathrm{L}$. & Banbotua & Chenopodiaceae & Herb & $\mathrm{C}$ \\
\hline 110 & Chrozophora plicata $\mathrm{L}$. & Khudiokra & Euphorbiaceae & Herb & $\mathrm{C}$ \\
\hline 111 & Chrysanthamum coronarium L. & Chandramollica & Asteraceae & Shrub & $\mathrm{C}$ \\
\hline 112 & Crysopogon aciculatus (Retz.) Trin. & Premkanta & Poaceae & Herb & $\mathrm{VC}$ \\
\hline 113 & Cicer arietinum $\mathrm{L}$. & Cholla & Fabaceae & Herb & $\mathrm{C}$ \\
\hline 114 & Cinnaтотит tamala Nees. & Tejpata & Lauraceae & Shrub & $\mathrm{C}$ \\
\hline 115 & Circium arvense (L.) Scop. & Circium & Asteraceae & Herb & $\mathrm{C}$ \\
\hline 116 & Citrullus lanatus (Thunb.) Mans. & Turmuz & Cucurbitaceae & Climber & $\mathrm{VC}$ \\
\hline 117 & Citrus aurantifolia $\mathrm{Sw}$. & Kagochilebu & Rutaceae & Shrub & $\mathrm{C}$ \\
\hline 118 & Citrus grandis (L.) Osbeck. & Jambura & Rutaceae & Tree & $\mathrm{C}$ \\
\hline 119 & Citrus limon (L.) Burm.f. & Goralebu & Rutaceae & Shrub & $\mathrm{C}$ \\
\hline 120 & Citrus reticulata Blanco & Kamlalebu & Rtaceae & Tree & VS \\
\hline 121 & Clausena heptaphylla W. \& A. & Panmashlla & Rutaceae & Shrub & ES \\
\hline 122 & Cleome viscosa $\mathrm{L}$. & Hurhuria & Capparaceae & Herb & $\mathrm{C}$ \\
\hline 123 & Clerodendrum viscosum Vent. & Bhat & Verbenaceae & Shrub & $\mathrm{VC}$ \\
\hline 124 & Clitoria tarnetea $\mathrm{L}$. & Aparajita & Fabaceae & Climber & $\mathrm{C}$ \\
\hline
\end{tabular}




\begin{tabular}{|c|c|c|c|c|c|}
\hline 125 & Coccinea cordifolia (L.) Cogn. & Telakucha & Cucurbitaceae & Climber & $\mathrm{VC}$ \\
\hline 126 & Cocos nucifera $\mathrm{L}$. & Narikel & Arecaceae & Tree & $\mathrm{VC}$ \\
\hline 127 & Codiaeum variegatum Bl. & Patabahar & Euphorbiaceae & Shrub & $\mathrm{VC}$ \\
\hline 128 & Colocasia esculenta (L.) Schott. & Kochu & Araceae & Herb & $\mathrm{VC}$ \\
\hline 129 & Commelina benghalensis L. & Kanchira & Commelinaceae & Herb & $\mathrm{C}$ \\
\hline 130 & Corchorus capsularis L. & Deshipat & Tiliaceae & Shrub & $\mathrm{VC}$ \\
\hline 131 & Coriandrum sativum $\mathrm{L}$. & Dhania & Apiaceae & Herb & $\mathrm{C}$ \\
\hline 132 & Cosmos caudatus Cav. & Cosmos & Asteraceae & Herb & $\mathrm{C}$ \\
\hline 133 & Couroupita guianensis Aubl. & Nagliggom & Lecythidaceae & Tree & VS \\
\hline 134 & Cresentia cujette $\mathrm{L}$. & Bleibel, duggugi & Bignoniaceae & Tree & VS \\
\hline 135 & Croton bonplandianum Bail. & Croton & Euphorbiaceae & Herb & $\mathrm{C}$ \\
\hline 136 & Cucumis melo $\mathrm{L}$. & Bangi & Cucurbitaceae & Climber & $\mathrm{C}$ \\
\hline 137 & Cucumis sativus L. & Sasha & Cucurbitaceae & Climber & $\mathrm{C}$ \\
\hline 138 & Cucumis callosus Cogn. & Bangumak & Cucurbitaceae & Climber & $\mathrm{C}$ \\
\hline 139 & Cucurbita maxima Duch. & Mistikumra & Cucurbitaceae & Climber & $\mathrm{C}$ \\
\hline 140 & Cucurbita pepo DC. & Mistikumra & Cucurbitaceae & Climber & $\mathrm{C}$ \\
\hline 141 & Cucurbita moschata Duch. & Mistikumra & Cucurbitaceae & Climber & $\mathrm{C}$ \\
\hline 142 & Costus speciosus $\mathrm{Sm}$. & Kushtha & Costaceae & Herb & $\mathrm{C}$ \\
\hline 143 & Curcuma longa $\mathrm{L}$. & Holud & Zingiberaceae & Herb & $\mathrm{C}$ \\
\hline 144 & Curcuma amada Roxb. & Amada & Zingiberaceae & Herb & ES \\
\hline 145 & Curcuma zeoderia Rosc. & Shathi & Zingiberaceae & Herb & $\mathrm{C}$ \\
\hline 146 & Cuscuta reflexa Roxb. & Sarnalata & Cuscutaceae & Climber & $\mathrm{C}$ \\
\hline 147 & Cymbopogon citratus (DC.) Stapf. & Lemongrass & Poaceae & Herb & $\mathrm{C}$ \\
\hline 148 & Cynodon dactylon Pers. & Durbaghas & Poaceae & Herb & $\mathrm{VC}$ \\
\hline 149 & Cyperus rotundus $\mathrm{L}$. & Muthaghas & Cyperaceae & Herb & $\mathrm{VC}$ \\
\hline 150 & Dahlia hybrida L. & Dahlia & Asteraceae & Herb & $\mathrm{C}$ \\
\hline 151 & Dalbergia sissoo Roxb. & Sissoo & Fabaceae & Tree & $\mathrm{VC}$ \\
\hline 152 & Datura metel L. & Dhutra & Solanaceae & Shrub & $\mathrm{C}$ \\
\hline 153 & Daucas carota $\mathrm{L}$. & Gajor & Apiaceae & Herb & $\mathrm{C}$ \\
\hline 154 & Delonix regia (Boj.) Raf. & Krisnachura & Caesalpiniaceae & Tree & $\mathrm{C}$ \\
\hline 155 & Derris indica (Lamk.) Benth. & Karanja & Fabaceae & Tree & $\mathrm{C}$ \\
\hline 156 & Desmodium triflorum (L.) DC. & Kodaliya & Fabaceae & Herb & $\mathrm{C}$ \\
\hline 157 & Digera muricata (L.) Mart. & Digera & Amaranthaceae & Herb & VS \\
\hline 158 & Dillenia indica $\mathrm{L}$. & Chalta & Dilleniaceae & Tree & $\mathrm{C}$ \\
\hline 159 & Dioscoria alata $\mathrm{L}$. & Chuprialu & Dioscoriaceae & Climber & VS \\
\hline 160 & Diplocyclos palmatus (L.) Jeffrey & Mala & Cucurbitaceae & Climber & VS \\
\hline 161 & Dyospyros perigrina (Gaertn.) Gur. & Gab & Ebenaceae & Tree & $\mathrm{C}$ \\
\hline 162 & Dyospyros philippensis (Des.) Gam. & Bilatigab & Ebenaceae & Tree & $\mathrm{C}$ \\
\hline 163 & Diospyros montana Roxb. & Tamal & Ebenaceae & Tree & VS \\
\hline 164 & Dracaena spicata Roxb. & Dracaena & Agavaceae & Herb & $\mathrm{C}$ \\
\hline 165 & Duranta repens $\mathrm{L}$. & Katamehedi & Verbenaceae & Shrub & $\mathrm{C}$ \\
\hline 166 & Eclipta alba (L.) Hassk. & Kalokeshi & Asteraceae & Herb & $\mathrm{C}$ \\
\hline 167 & Eichhornia crassipes (Mart.) Sol.-Lau. & Kochuripana & Pontedariaceae & Herb & $\mathrm{C}$ \\
\hline 168 & Elaeocarpus robustus Roxb. & Jalpai & Elaeocarpaceae & Tree & $\mathrm{C}$ \\
\hline 169 & Elusine indica (L.) Gaertn & Malankuri & Poaceae & Herb & $\mathrm{C}$ \\
\hline 170 & Enhydra flactuans Lour. & Helencha & Asteraceae & Herb & VS \\
\hline 171 & Erythrina variegata $\mathrm{L}$. & Madar & Fabaceae & Tree & $\mathrm{C}$ \\
\hline 172 & Ethulia conyzoides $\mathrm{L}$. & Golphuli & Asteraceae & Herb & VS \\
\hline 173 & Eucalyptus citrodora Hook. & Eucalyptus & Myrtaceae & Tree & $\mathrm{C}$ \\
\hline 174 & Eupatorium odoratum L. & Germanlata & Asteraceae & Shrub & $\mathrm{C}$ \\
\hline 175 & Euphorbia hirta L. & Dudhiya & Euphorbiaceae & Herb & $\mathrm{VC}$ \\
\hline 176 & Euphorbia pulcherrima Willd. & Lalpata & Euphorbiaceae & Shrub & $\mathrm{C}$ \\
\hline 177 & Euphorbia thymifolia Burm.f. & Dudhia & Euphorbiaceae & Herb & $\mathrm{C}$ \\
\hline 178 & Exacum pedunculatum Roxb. & Kachuri & Gentianaceae & Herb & $\mathrm{C}$ \\
\hline 179 & Evolvulus numularius L. & Bhuiokra & Convolvulaceae & Herb & $\mathrm{VC}$ \\
\hline 180 & Feronia limonia (L.) Sw. & Kothbel & Rutaceae & Tree & $\mathrm{C}$ \\
\hline 181 & Ficus benghalensis L. & Bot & Moraceae & Tree & $\mathrm{VC}$ \\
\hline 182 & Ficus elastica Roxb. & Bharotiorubber & Moraceae & Tree & $\mathrm{C}$ \\
\hline 183 & Ficus hispida L.f. & Kakdumur & Moraceae & Tree & $\mathrm{C}$ \\
\hline 184 & Ficus racemosa $\mathrm{L}$. & Jogadumur & Moraceae & Tree & $\mathrm{C}$ \\
\hline 185 & Ficus religiosa $\mathrm{L}$. & Pakur & Moraceae & Tree & $\mathrm{C}$ \\
\hline 186 & Flacourtia indica (Burm. f.) Merr. & Bichuti & Flacourtiaceae & Shrub & $\mathrm{C}$ \\
\hline 187 & Fumaria indica (Hassk.) Pugs. & Bassulpha & Fumariaceae & Herb & $\mathrm{C}$ \\
\hline 188 & Gardenia jasminoides Ellis. & Gandharaj & Rubiaceae & Shrub & $\mathrm{C}$ \\
\hline 189 & Glinus oppositifolius (L.) A.DC. & Gima & Molluginaceae & Herb & VS \\
\hline 190 & Gloriosa superba L. & Ulatchandal & Liliaceae & Herb & ES \\
\hline 191 & Glycine max (L.) Merr. & Soyabeen & Fabaceae & Shrub & $\mathrm{C}$ \\
\hline 192 & Glycosmis pentaphylla (Retz.) A. DC. & Datmajan & Rutaceae & Shrub & $\mathrm{C}$ \\
\hline 193 & Gnaphalium pulvinatum DC. & Barakamra & Asteraceae & Herb & $\mathrm{VC}$ \\
\hline 194 & Gnaphalium indicum L. & Kamra & Asteraceae & Herb & $\mathrm{VC}$ \\
\hline 195 & Gossypium harbaceum L. & Karpas & Malvaceae & Shrub & $\mathrm{C}$ \\
\hline 196 & Grangea maderaspatana Poir. & Nemuti & Asteraceae & Herb & $\mathrm{C}$ \\
\hline 197 & Gymnopetalum cochinchinense (Lour.) Kurj. & Kabuti & Cucurbitaceae & Climber & VS \\
\hline
\end{tabular}




\begin{tabular}{|c|c|c|c|c|c|}
\hline 198 & Helianthus annuиs $\mathrm{L}$. & Surjamukhi & Asteraceae & Herb & $\overline{\mathrm{VC}}$ \\
\hline 199 & Heliotropium indicum $\mathrm{L}$. & Hatisur & Boraginaceae & Herb & $\mathrm{VC}$ \\
\hline 200 & Heliconia rostrata Ruiz \& Pav. & Heliconia & Heliconiaceae & Herb & $\mathrm{C}$ \\
\hline 201 & Hemidesmus indicus $\mathrm{Br}$. & Anantamul & Asclepiadaceae & Climber & $\mathrm{C}$ \\
\hline 202 & Heriteira fomes Buch-Ham. & Sundari & Sterculiaceae & Tree & VS \\
\hline 203 & Hibiscus esculentus L. & Bhindi & Malvaceae & Herb & $\mathrm{VC}$ \\
\hline 204 & Hibiscus mutabilis L. & Sthalpadma & Malvaceae & Shrub & $\mathrm{C}$ \\
\hline 205 & Hibiscus rosa-sinensis $\mathrm{L}$. & Jaba & Malvaceae & Shrub & $\mathrm{VC}$ \\
\hline 206 & Hygrophila auriculata (K.Schum.) Heine & Talmakhna & Acanthaceae & Herb & $\mathrm{C}$ \\
\hline 207 & Hydrilla verticillata (L. f.) Royle & Kureli & Hydrocharitaceae & Herb & $\mathrm{C}$ \\
\hline 207 & Ichnocarpus frutescens (L.) R.Br. & Loilata & Apocynaceae & Climber & $\mathrm{C}$ \\
\hline 209 & Impatiens balsamina $\mathrm{L}$. & Dopati & Balsaminaceae & Herb & $\mathrm{C}$ \\
\hline 210 & Indigofera tinctoria $\mathrm{L}$. & Nil & Fabaceae & Shrub & VS \\
\hline 211 & Ipomoea aquatica Forsk & Kalmi & Convolvulaceae & Climber & $\mathrm{C}$ \\
\hline 212 & Ipomoea batatus $\mathrm{L}$. & Mistialu & Convolvulaceae & Climber & $\mathrm{C}$ \\
\hline 213 & Ipomoea fistulosa Mart. Ex Choisy & Dholkalmi & Convolvulaceae & Shrub & $\mathrm{C}$ \\
\hline 214 & Ixora coccinea $\mathrm{L}$. & Rangan & Rubiaceae & Shrub & $\mathrm{C}$ \\
\hline 215 & Jacaranda mimosifolia D. Don. & Jacaranda & Bignoniaceae & Tree & VS \\
\hline 216 & Jasminum sambac (L.) Ait. & Beli & Oleaceae & Shrub & $\mathrm{C}$ \\
\hline 217 & Jatropha curcas L. & Jamalgota & Euphorbiaceae & Shrub & $\mathrm{C}$ \\
\hline 218 & Jatropha gossypifolia $\mathrm{L}$. & Lalverenda & Euphorbiaceae & Shrub & $\mathrm{C}$ \\
\hline 219 & Justicia gendarussa $\mathrm{L}$. & Jagthmadan & Acanthaceae & Shrub & $\mathrm{VC}$ \\
\hline 220 & Kalanchoe pinnata (Lam.) Pers. & Pathorkuchi & Crassulaceae & Herb & $\mathrm{C}$ \\
\hline 221 & Lablab purpureus (L.) Sweet. & Sim & Fabaceae & Climber & $\mathrm{VC}$ \\
\hline 222 & Lactuca sativa $\mathrm{L}$. & Lettuce & Asteraceae & Herb & $\mathrm{C}$ \\
\hline 223 & Lagenaria siceraria (Mol.) Stan. & Lau & Cucurbitaceae & Climber & $\mathrm{VC}$ \\
\hline 224 & Lagerstroemia speciosa (L.) Pers. & Jarul & Lythraceae & Tree & $\mathrm{VC}$ \\
\hline 225 & Lannea coromandelica (Houtt.) Merr. & Jiga & Anacardiaceae & Tree & VS \\
\hline 226 & Lantana camara $\mathrm{L}$. & Lantana & Verbenaceae & Shrub & $\mathrm{C}$ \\
\hline 227 & Lathyrus sativus $\mathrm{L}$. & Kheshari & Fabaceae & Herb & $\mathrm{C}$ \\
\hline 228 & Launaea asplenifolia $\mathrm{Hk} . \mathrm{f}$. & Tikdana & Asteraceae & Herb & $\mathrm{C}$ \\
\hline 229 & Lawsonia inermis $\mathrm{L}$. & Mehedi & Lythraceae & Shrub & $\mathrm{C}$ \\
\hline 230 & Leea macrophylla Roxb. & Hastikalash & Leeaceae & Herb & VS \\
\hline 231 & Lemna perpusilla Torr. & Khudipana & Lemnaceae & Herb & $\mathrm{C}$ \\
\hline 232 & Lens culinaris Medik. & Masur & Fabaceae & Herb & $\mathrm{C}$ \\
\hline 233 & Leonurus sibiricus $\mathrm{L}$. & Roktodron & Lamiaceae & Shrub & $\mathrm{C}$ \\
\hline 234 & Leucas aspera $\mathrm{L}$. & Setodron & Lamiaceae & Herb & $\mathrm{C}$ \\
\hline 235 & Leucas cephalotes (Roth.) Spreng. & Dandakolos & Lamiaceae & Herb & $\mathrm{C}$ \\
\hline 236 & Lippia nodiflora (L.) Rich. & Bhuiokra & Verbenaceae & Herb & $\mathrm{VC}$ \\
\hline 237 & Lippia alba $\mathrm{L}$. & Borobhuiokra & Verbenaceae & Shrub & $\mathrm{C}$ \\
\hline 238 & Litchi chinensis Sonn. & Lichu & Sapindaceae & Tree & $\mathrm{VC}$ \\
\hline 239 & Litsea monopetala (Roxb.) Pers. & Pipulti & Lauraceae & Shrub & $\mathrm{C}$ \\
\hline 240 & Loranthus longifolius Desr. & Bhanda & Loranthaceae & Herb & $\mathrm{C}$ \\
\hline 241 & Ludwigia adscendens (L.) Hara. & Kesordam & Onagraceae & Herb & $\mathrm{C}$ \\
\hline 242 & Luffa acutangula Roxb. & Jhinga & Cucurbitaceae & Climber & $\mathrm{C}$ \\
\hline 243 & Luffa cylindrica (L.) Roem. & Dhundal & Cucurbitaceae & Climber & $\mathrm{C}$ \\
\hline 244 & Lycopersicon lycopersicum (L.) Karst. & Tomato & Solanaceae & Herb & $\mathrm{VC}$ \\
\hline 245 & Madhuca indica Gmell. & Mahua & Sapotaceae & Tree & $\mathrm{C}$ \\
\hline 246 & Magnolia grandiflora $\mathrm{L}$. & Udaypadma & Magnoliaceae & Tree & $\mathrm{C}$ \\
\hline 247 & Mangifera indica $\mathrm{L}$. & $\mathrm{Am}$ & Anacardiaceae & Tree & $\mathrm{VC}$ \\
\hline 248 & Manihot esculenta Crantz. & Kasava & Euphorbiaceae & Shrub & $\mathrm{C}$ \\
\hline 249 & Manilkara achras (Mill.) Per. & Sofeda & Sapotaceae & Tree & $\mathrm{C}$ \\
\hline 250 & Marselia quadrifoliata $\mathrm{L}$. & Sushnishak & Marsileaceae & Herb & $\mathrm{C}$ \\
\hline 251 & Melia sempervirens (L.) All. & Ghoraneem & Meliaceae & Tree & $\mathrm{C}$ \\
\hline 252 & Melilotus alba Lamk. & Sadamethi & Fabaceae & Herb & $\mathrm{C}$ \\
\hline 253 & Melothria maderaspatana Cogn. & Agmukhi & Cucurbitaceae & Climber & VS \\
\hline 254 & Mentha arvensis $\mathrm{L}$. & Pudina & Lamiaceae & Herb & VS \\
\hline 255 & Mesua nagassarium (Burm.f.) Kost. & Nageswar & Clusiaceae & Tree & $\mathrm{C}$ \\
\hline 256 & Michelia champaca $\mathrm{L}$. & Champa & Magnoliaceae & Tree & $\mathrm{C}$ \\
\hline 257 & Mikania cordata (Burm.f.) Roxb. & Asamlata & Asteraceae & Climber & $\mathrm{C}$ \\
\hline 258 & Mimosa pudica $\mathrm{L}$. & Lajjabati & Mimosaceae & Climber & $\mathrm{C}$ \\
\hline 259 & Mimuspos elengi $\mathrm{L}$. & Bokul & Sapotaceae & Tree & $\mathrm{C}$ \\
\hline 260 & Mirabilis jalapa $\mathrm{L}$. & Sandhamoni & Nyctaginaceae & Herb & $\mathrm{C}$ \\
\hline 261 & Mollugo pentaphylla $\mathrm{L}$. & Khetpapra & Molluginaceae & Herb & $\mathrm{C}$ \\
\hline 262 & Momordica charantea $\mathrm{L}$. & Korola & Cucurbitaceae & Climber & $\mathrm{VC}$ \\
\hline 263 & Momordica cochinchinensis Spreng & Kakrol & Cucurbitaceae & Climber & $\mathrm{C}$ \\
\hline 264 & Momordica dioica Roxb. & Ghekorola & Cucurbitaceae & Climber & VS \\
\hline 265 & Monochoria hastata (L.) Solms. & Baranukha & Pontenderiaceae & Herb & $\mathrm{C}$ \\
\hline 266 & Monochoria vaginalis (Burm. f.) Presl. & Sarkachu & Pontenderiaceae & Herb & $\mathrm{C}$ \\
\hline 267 & Moringa oleifera Lamk. & Sajna & Moringaceae & Tree & $\mathrm{VC}$ \\
\hline 268 & Morus indica $\mathrm{L}$. & Tut & Moraceae & Shrub & $\mathrm{C}$ \\
\hline 269 & Murraya koenigii Spreng. & Curryleaf & Rutaceae & Tree & VS \\
\hline 270 & Murraya paniculata (L.) Jacq. & Kamini & Rutaceae & Shrub & $\mathrm{VC}$ \\
\hline 271 & Musa sapientum $\mathrm{L}$. & Kala & Musaceae & Shrub & $\mathrm{VC}$ \\
\hline
\end{tabular}




\begin{tabular}{|c|c|c|c|c|c|}
\hline 272 & Mussaenda erythrophylla Schum. \& Thon. & Mucenda & Rubiaceae & Shrub & $\mathrm{VC}$ \\
\hline 273 & Najas graminea Del. & Najas & Najadaceae & Herb & $\mathrm{C}$ \\
\hline 274 & Nerium indicum $\mathrm{L}$. & Karabi & Apocynaceae & Shrub & $\mathrm{C}$ \\
\hline 275 & Nicotiana plumbaginifolia Viv. & Bantamak & Solanaceae & Herb & $\mathrm{VC}$ \\
\hline 276 & Nicotiana tabacum L. & Tamak & Solanaceae & Herb & $\mathrm{C}$ \\
\hline 277 & Nyctanthes arbortristis L. & Sheuli & Verbenaceae & Shrub & $\mathrm{C}$ \\
\hline 278 & Nymphaea nouchali Burm.f. & Shapla & Nymphaeaceae & Herb & $\mathrm{C}$ \\
\hline 279 & Nymphoides indicum (L.)Kuntz. & Panchuli & Menyanthaceae & Herb & $\mathrm{C}$ \\
\hline 280 & Ocimum basilicum $\mathrm{L}$. & Babuitulsi & Lamiaceae & Shrub & $\mathrm{C}$ \\
\hline 281 & Ocimum sanctum $\mathrm{L}$. & Tulsi & Lamiaceae & Shrub & $\mathrm{C}$ \\
\hline 282 & Oplismenus compositus (L.) P. Baeuv. & Gohur & Poaceae & Herb & $\mathrm{VC}$ \\
\hline 283 & Oryza sativa $\mathrm{L}$. & Dhan & Poaceae & Herb & $\mathrm{VC}$ \\
\hline 284 & Ottelia alismoides (L.)Pers. & Panikala & Hydrocharitaceae & Herb & $\mathrm{C}$ \\
\hline 285 & Oxalis corniculata $\mathrm{L}$. & Amrul & Oxalidaceae & Herb & $\mathrm{VC}$ \\
\hline 286 & Oxalis rubra $\mathrm{L}$. & Boroamrul & Oxalidaceae & Herb & $\mathrm{C}$ \\
\hline 287 & Paederia foetida $\mathrm{L}$. & Gandhavaduli & Rubiaceae & Shrub & VS \\
\hline 288 & Panicum repens $\mathrm{L}$. & Dhanighas & Poaceae & Herb & $\mathrm{C}$ \\
\hline 289 & Parthenium hysterophorus L. & Gandiboti & Asteraceae & Shrub & $\mathrm{VC}$ \\
\hline 290 & Passiflora foetida $\mathrm{L}$. & Jhumkolata & Passifloraceae & Climber & VS \\
\hline 291 & Peltophorum pterocarpum (DC.) Baker ex Heyne & Holudkrisnachura & Caesalpiniaceae & Tree & $\mathrm{C}$ \\
\hline 292 & Peperomia pellucida Kunth. & Peperomia & Piperaceae & Herb & $\mathrm{C}$ \\
\hline 293 & Petunia hybrida L. & Petunia & Solanaceae & Herb & $\mathrm{C}$ \\
\hline 294 & Phaseolus mungo $\mathrm{L}$. & Mashkalai & Fabaceae & Herb & $\mathrm{C}$ \\
\hline 295 & Phoenix sylvestris (L.) Roxb. & Khejur & Arecaceae & Tree & $\mathrm{VC}$ \\
\hline 296 & Phyllanthus emblica L. & Amloki & Euphorbiaceae & Tree & $\mathrm{C}$ \\
\hline 297 & Phyllanthus niruri $\mathrm{L}$. & Bhuiamla & Euphorbiaceae & Herb & $\mathrm{C}$ \\
\hline 298 & Phyllanthus reticulatus Poir. & Chitki & Euphorbiaceae & Shrub & $\mathrm{C}$ \\
\hline 299 & Phyllanthus urinaria $\mathrm{L}$. & Amla & Euphorbiaceae & Herb & $\mathrm{C}$ \\
\hline 300 & Physalis minima $\mathrm{L}$. & Kapalphutki & Solanaceae & Herb & $\mathrm{C}$ \\
\hline 301 & Piper betel L. & Pan & Piperaceae & Climber & $\mathrm{C}$ \\
\hline 302 & Piper nigrum $\mathrm{L}$. & Golmorich & Piperaceae & Climber & VS \\
\hline 303 & Pistia strateotes $\mathrm{L}$. & Topapana & Araceae & Herb & $\mathrm{C}$ \\
\hline 304 & Pisum sativum $\mathrm{L}$. & Motor & Fabaceae & Herb & $\mathrm{C}$ \\
\hline 305 & Pithecellobium dulce (Roxb.) Benth & Jilapiphol & Mimosaceae & Tree & $\mathrm{C}$ \\
\hline 306 & Plumeria acutifolia $\mathrm{L}$. & Kathgolap & Apocynaceae & Tree & $\mathrm{C}$ \\
\hline 307 & Plumeria rubra $\mathrm{L}$ & Lalkathgolap & Apocynaceae & Tree & $\mathrm{C}$ \\
\hline 308 & Polyalthia longifolia (Sonn.) Thw. & Raganigandha & Annonaceae & Herb & $\mathrm{C}$ \\
\hline 309 & Polygonum barbatum L. & Panimorich & Polygonaceae & Herb & $\mathrm{C}$ \\
\hline 310 & Polygonum hydropiper $\mathrm{L}$. & Panimorich & Polygonaceae & Herb & $\mathrm{C}$ \\
\hline 311 & Polygonum orientale L. & Boropanimorich & Polygonaceae & Shrub & $\mathrm{C}$ \\
\hline 312 & Polygonum plebejum $\mathrm{Br}$. & Panimorich & Polygonaceae & Herb & $\mathrm{C}$ \\
\hline 313 & Portulaca oleracea L. & Baranunia & Portulacaceae & Herb & $\mathrm{C}$ \\
\hline 314 & Portulaca quadrifida $\mathrm{L}$. & Chotonunia & Portulacaceae & Herb & $\mathrm{C}$ \\
\hline 315 & Potamogeton mucronatus Presl. & & Potamogetonaceae & Herb & $\mathrm{C}$ \\
\hline 316 & Psidium guajava (L.) Bat. & Piyara & Myrtaceae & Tree & VC \\
\hline 317 & Psoralea corylifolia L. & Buchikdana & Fabaceae & Shrub & $\mathrm{C}$ \\
\hline 318 & Punica granatum $\mathrm{L}$. & Dalim & Punicaceae & Tree & $\mathrm{C}$ \\
\hline 319 & Quisqualis indica $\mathrm{L}$. & Madhabilata & Combretaceae & Shrub & $\mathrm{C}$ \\
\hline 320 & Ranunculus scleratus $\mathrm{L}$. & Podika & Ranunculaceae & Herb & $\mathrm{C}$ \\
\hline 321 & Raphanus sativus L. & Mula & Brasssicaceae & Herb & $\mathrm{VC}$ \\
\hline 322 & Rauvolfia serpentina Benth. & Sarpagandha & Apocynaceae & Herb & VS \\
\hline 323 & Rosa damascena Mill. & Kanta golap & Rosaceae & Shrub & $\mathrm{VC}$ \\
\hline 324 & Ravenala madagascariensis Gmell. & Panthapadap & Strelitziaceae & Shrub & $\mathrm{C}$ \\
\hline 325 & Ricinus communis L. & Bherenda & Euphorbiaceae & Shrub & $\mathrm{C}$ \\
\hline 326 & Rosa centifolia L. & Golap & Rosaceae & Shrub & $\mathrm{VC}$ \\
\hline 327 & Ruellia tuberosa $\mathrm{L}$. & Chatpotey & Acanthaceae & Herb & $\mathrm{C}$ \\
\hline 328 & Rumex maritimus L. & Banpalong & Polygonaceae & Herb & $\mathrm{VC}$ \\
\hline 329 & Saccharum officinarum L. & Akh & Poaceae & Shrub & $\mathrm{VC}$ \\
\hline 330 & Saccharum spontaneum L. & Kash & Poaceae & Shrub & $\mathrm{C}$ \\
\hline 331 & Sagittaria sagitifolia $\mathrm{L}$. & Muyamuya & Alismataceae & Herb & $\mathrm{C}$ \\
\hline 332 & Salvia plebeja $\mathrm{Br}$. & Bhuitulsi & Lamiaceae & Herb & $\mathrm{C}$ \\
\hline 333 & Samanea saman (Jacq.) Merr. & Rain tree & Fabaceae & Tree & $\mathrm{C}$ \\
\hline 334 & Saraca indica $\mathrm{L}$. & Asoke & Caesalpiniaceae & Tree & $\mathrm{C}$ \\
\hline 335 & Scirpus grossus L. & Kesur & Cyperaceae & Climber & $\mathrm{C}$ \\
\hline 336 & Scirpus articulates (L.) Palla. & Chechur & Cyperaceae & Herb & $\mathrm{C}$ \\
\hline 337 & Scoparia dulcis $\mathrm{L}$. & Bandhunay & Scrophulariaceae & Herb & $\mathrm{VC}$ \\
\hline 338 & Sesamum indicum $\mathrm{L}$. & Til & Pedaliaceae & Herb & $\mathrm{VC}$ \\
\hline 339 & Sesbania canabina Pers. & Dhanchi & Fabaceae & Shrub & $\mathrm{C}$ \\
\hline 340 & Sesbania grandiflora (L.) Pers. & Bakphul & Fabaceae & Tree & $\mathrm{C}$ \\
\hline 341 & Sida acuta Burm. & Berela & Malvaceae & Herb & $\mathrm{C}$ \\
\hline 342 & Sida cordata (Burm.f.) Borss. & Pitberela & Malvaceae & Herb & $\mathrm{C}$ \\
\hline 343 & Smilax zeylanica $\mathrm{L}$. & Kumarilata & Smilaceae & Climber & VS \\
\hline 344 & Solanum filisifolium Ort. & Titbegun & Solanaceae & Shrub & $\mathrm{C}$ \\
\hline
\end{tabular}




\begin{tabular}{|c|c|c|c|c|c|}
\hline 345 & Solanum melongena Wall. & Begun & Solanaceae & Shrub & $\mathrm{VC}$ \\
\hline 346 & Solanum nigrum L. & Kakmachi & Solanaceae & Herb & $\mathrm{VC}$ \\
\hline 347 & Solanum surattense Burm.f. & Kantakini & Solanaceae & Herb & $\mathrm{C}$ \\
\hline 348 & Solanum torvum $\mathrm{Sw}$. & Gothbegun & Solanaceae & Shrub & $\mathrm{C}$ \\
\hline 349 & Solanum tuberosum L. & Alu & Solanaceae & Herb & $\mathrm{VC}$ \\
\hline 350 & Sonchus arvensis L. & Banpalang & Asteraceae & Herb & $\mathrm{C}$ \\
\hline 351 & Sonchus asper L. & Banpalong & Asteraceae & Herb & $\mathrm{C}$ \\
\hline 352 & Spilanthes acmella L. & Marhatitiga & Asteraceae & Herb & $\mathrm{C}$ \\
\hline 353 & Spirodela polyrhiza (L.) Schleid. & Spirodela & Lemnaceae & Herb & $\mathrm{C}$ \\
\hline 354 & Spondias dulcis Sol. ex Park. & Bilatiamra & Anacardiaceae & Tree & $\mathrm{C}$ \\
\hline 355 & Spondias pinnata (L.f.) Kurz. & Deshiamra & Anacardiaceae & Tree & VS \\
\hline 356 & Stephania japonica (Thunb.) Miers. & Aknadi & Menispermaceae & Climber & $\mathrm{C}$ \\
\hline 357 & Streblus asper Lour. & Shaora & Moraceae & Tree & VS \\
\hline 358 & Swietenia macrophylla King. & Boromehagoni & Meliaceae & Tree & $\mathrm{VC}$ \\
\hline 359 & Swietenia mahagoni (L.) Jacq. & Mahagoni & Meliaceae & Tree & $\mathrm{C}$ \\
\hline 360 & Synedrella nodiflora (L.) Gaertn. & Marhatitiga & Asteraceae & Herb & $\mathrm{C}$ \\
\hline 361 & Syzygium cumini (L.) Skeel. & Jam & Myrtaceae & Tree & $\mathrm{VC}$ \\
\hline 362 & Syzygium fruticosum (Roxb.) DC. & Khudijam & Myrtaceae & Tree & $\mathrm{C}$ \\
\hline 363 & Syzygium jambos (L.) Alston. & Golapjam & Myrtaceae & Tree & VS \\
\hline 364 & Syzygium samarangense (B1.) Merr. \& Perry. & Jamrul & Myrtaceae & Tree & $\mathrm{C}$ \\
\hline 365 & Tabebuia rosea (Bertol.) DC. & Tabebuia & Bignoniaceae & Tree & ES \\
\hline 366 & Tagetes erecta $\mathrm{L}$. & Gadaphul & Asteraceae & Herb & $\mathrm{C}$ \\
\hline 367 & Tagetes patula $\mathrm{L}$. & Genda & Asteraceae & Herb & $\mathrm{VC}$ \\
\hline 368 & Thladiantha cordifilia (B1.) Cogn. & & Cucurbitaceae & Climber & VS \\
\hline 369 & Tamarindus indica $\mathrm{L}$. & Tentul & Caesalpiniaceae & Tree & $\mathrm{C}$ \\
\hline 370 & Tecoma stans (L.) H.B.\&K. & Sonapati & Bignoniaceae & Tree & VS \\
\hline 371 & Tectona grandis L.f. & Segun & Verbenaceae & Tree & $\mathrm{C}$ \\
\hline 372 & Terminalia arjuna (Roxb.) Wt.\& Arn. & Arjun & Combretaceae & Tree & $\mathrm{C}$ \\
\hline 373 & Terminalia belerica Roxb. & Bohera & Combretaceae & Tree & VS \\
\hline 374 & Terminalia catappa $\mathrm{L}$. & Kathbadam & Combretaceae & Tree & $\mathrm{C}$ \\
\hline 375 & Terminalia chebula (Gaertn.) Retz. & Haritaki & Combretaceae & Tree & $\mathrm{C}$ \\
\hline 376 & Thespesia populnea (L.) Sol. ex Corr. & Parashpipul & Malvaceae & Tree & $\mathrm{C}$ \\
\hline 377 & Thevetia peruviana (Pers.) K. Schum. & Haldekorobi & Apocynaceae & Tree & $\mathrm{C}$ \\
\hline 378 & Thunbergia grandiflora Roxb. & Nillata & Acanthaceae & Climber & VS \\
\hline 379 & Tinospora cordifolia (Willd.) Hook.f. & Gulancha & Menispermaceae & Climber & $\mathrm{C}$ \\
\hline 380 & Toona ciliata J.Roem. & Toon & Meliaceae & Tree & VS \\
\hline 381 & Tragia involucrata $\mathrm{L}$. & Bichuti & Euphorbiaceae & Herb & VS \\
\hline 382 & Trapa bispinosa Roxb. & Paniphal & Trapaceae & Herb & $\mathrm{C}$ \\
\hline 383 & Trema orientalis (L.) Bl. & Jibon & Ulmaceae & Tree & VS \\
\hline 384 & Trewia polycarpa Benth. & Batul & Euphorbiaceae & Tree & $\mathrm{C}$ \\
\hline 385 & Trichosanthes anguina $\mathrm{L}$. & Chichinga & Cucurbitaceae & Climber & $\mathrm{C}$ \\
\hline 386 & Trichosanthes bracteata (Lamk.) Voigt. & Makal & Cucurbitaceae & Climber & VS \\
\hline 387 & Trichosanthes cordata Roxb. & Bhuikakra & Cucurbitaceae & Climber & $\mathrm{C}$ \\
\hline 388 & Trichosanthes dioica Roxb. & Potol & Cucurbitaceae & Climber & $\mathrm{C}$ \\
\hline 389 & Trichosanthes cucumerina $\mathrm{L}$. & Banchichinga & Cucurbitaceae & Climber & $\mathrm{C}$ \\
\hline 390 & Tridax procumbens $\mathrm{L}$. & Tridhara & Asteraceae & Herb & $\mathrm{VC}$ \\
\hline 391 & Triticum aestivum $\mathrm{L}$. & Gom & Poaceae & Herb & $\mathrm{VC}$ \\
\hline 392 & Typha elephantiana Roxb. & Hogla & Typhaceae & Shrub & VS \\
\hline 393 & Typhonium trilobatum (L.) Schott. & Ghetkachu & Araceae & Herb & $\mathrm{C}$ \\
\hline 394 & Uraria picta Desv. & Sankarjata & Fabaceae & Herb & $\mathrm{C}$ \\
\hline 395 & Urena lobata $\mathrm{L}$. & Banokra & Fabaceae & Herb & $\mathrm{C}$ \\
\hline 396 & Utricularia aurea Lour. & Jhangi & Lentibulariaceae & Herb & VS \\
\hline 397 & Vallisneria spiralis $\mathrm{L}$. & Potseola & Hydrocharitaceae & Herb & VS \\
\hline 398 & Vanda roxburghii R. Br. & Rasna & Orchidaceae & Herb & VS \\
\hline 399 & Vernonia patula (Dryand.) Merril. & Kuksim & Asteraceae & Herb & $\mathrm{C}$ \\
\hline 400 & Vernonia cineria (Roxb.) Less. & Kuksim & Asteraceae & Herb & $\mathrm{C}$ \\
\hline 401 & Vetivaria zizanioides (L.) Nash. & Benna & Poaceae & Herb & $\mathrm{C}$ \\
\hline 402 & Vicia faba $\mathrm{L}$. & Barasim & Fabaceae & Herb & $\mathrm{C}$ \\
\hline 403 & Vicia hirsuta Coch. & Masurchana & Fabaceae & Herb & $\mathrm{C}$ \\
\hline 404 & Vicia sativa $\mathrm{L}$. & Ankari & Fabaceae & Herb & $\mathrm{C}$ \\
\hline 405 & Vigna mungo (L.) Hepper & Mashkalai & Fabaceae & Herb & $\mathrm{C}$ \\
\hline 406 & Vigna radiata (L.) Wilezck. & Moog & Fabaceae & Herb & $\mathrm{C}$ \\
\hline 407 & Vigna sinensis Endl. Ex Hassk. & Borboti & Fabaceae & Climber & $\mathrm{C}$ \\
\hline 408 & Vitex negundo L. & Nishinda & Verbenaceae & Shrub & $\mathrm{C}$ \\
\hline 409 & Vitis quadrangularis Wall. & Harjora & Vitaceae & Climber & VS \\
\hline 410 & Vitis trifolia (L.) Don. & Banangur & Vitaceae & Climber & $\mathrm{C}$ \\
\hline 411 & Vitis vinifera $\mathrm{L}$. & Angur & Vitaceae & Climber & VS \\
\hline 412 & Webera campaniflora Hook. & Kakra & Rutaceae & Tree & VS \\
\hline 413 & Wedelia chinensis (Osb.) Merr. & Mohavringaraj & Asteraceae & Herb & $\mathrm{C}$ \\
\hline 414 & Wedelia trilobata (L.) Hitchc. & Mohavringaraj & Asteraceae & Herb & $\mathrm{VC}$ \\
\hline 415 & Withania somnifera Dunal. & Ashagandha & Solanaceae & Shrub & ES \\
\hline 416 & Wolffia arrhiza (L.) Horkel. & Guripana & Lemnaceae & Herb & $\mathrm{C}$ \\
\hline 417 & Xanthium indicum Koinig. & Ghagra & Asteraceae & Herb & $\mathrm{VC}$ \\
\hline 418 & Xylia dolabiformis Benth. & Lohakat & Mimosaceae & Tree & ES \\
\hline
\end{tabular}




\begin{tabular}{lllll}
\hline 419 & Youngia japonica (L.) DC. & Oochunti & Asteraceae & Herb \\
420 & Zea mays L. & Bhutta & Poaceae & Shrub \\
421 & Zeuxine sulcata Lindl. & Swethuli & Orchidaceae & Herb \\
422 & Zingiber officinale Rosc. & Ada & Zingiberaceae & Herb \\
423 & Zinnia elegans L. & Zinnia & Asteraceae & Herb \\
424 & Zizyphus mauritiana Lamk. & Kul, Boroi & Rhamnaceae & Tree \\
425 & Zizyphus oenoplea (L.) Mill. & Banboroi & Rhamnaceae & Tree \\
\hline
\end{tabular}

$\mathrm{VC}=$ Very common, $\mathrm{C}=$ Common, $\mathrm{VS}=$ Vulnerable species, $\mathrm{ES}=$ Endangered species

\section{Conclusion}

The present article focuses on an inventory of angiospermic flora of Rajshahi district. In this preliminary assessment, a total of 425 angiosperm species under 321 genera and 108 families were recorded. Habit analysis shows that herbs, shrubs, climbers and trees are represented by $186,74,52$ and 113 species, respectively. Out of the total number of species 281 were commom, 76 were very common, 57 were vulnerable and 11 were endangered species in the study area.

\section{Acknowledgements}

The author is grateful to the Ministry of Science, Information and Communication Technology, Government People's Republic of Bangladesh for financial support to complete this research work.

\section{References}

[1] Ahmed Z U, Begum Z N T, Hassan M A, Khondker M, Kabir S M H, Ahmad M, Ahmed A T A,Rahman A K A and Haque E U(Eds). Encyclopedia of Flora and Fauna of Bangladesh.. Angiosperms; Dicotyledons. Asiat. Soc.Bangladesh, Dhaka. Vol 6-12, 2007-2009.

[2] BBS (Bangladesh Bureau of Statistics).Statistical Year Book of Bangladesh, $23^{\text {rd }}$ edition, Bangladesh Bureau of Statistics, Planning Division, Ministry of Planning Government of Peoples Republic of Bangladesh, Dhaka, 2009.

[3] Cronquist A. An Integrated System of Classification of Flowering Plants. Columbia University Press. New York, 1981.

[4] Hooker JD. Flora of British India. L. Reeve and Co. Ltd. London, Vol 1-7, 1961.

[5] Huq AM. Plant Names of Bangladesh. Bangladesh National Herbarium, BARC, Dhaka, Bangladesh. 1986; pp.1-289.

[6] Khan MS, Rahman MM, Huq AM, Mia MMK and Hassan MA. Assessment of Biodiversity of Teknaf Game Reserve in Bangladesh focusing on economically and ecologically important plant species. Bangladesh J. Plant Taxon.1994; 1(1): 21-23.

[7] Khan MS and Huq AM. The vascular flora of Chunati wildlife Sanctuary in South Chittagong. Bangladesh J. Plant Taxon. 2001; 8(1): 47-64.
[8] Kirtikar KR and Basu BD. Indian Medicinal Plants. Lalit Mohan Basu, Alhabad, India. Vol 1-4, 1987.

[9] Pasha MK and Uddin SB. Dictionary of Plant Names of Bangladesh (Vascular Plants). Janokalyan Prokashani. Chittagong, Dhaka, Bangladesh. 2013; pp. 1-434.

[10] Prain D. Bengal Plants. Botanical Survey of India, Calcutta. India. Vol 1-2, 1963.

[11] Rahman AHMM, Islam AKMR and Zaman ATMN. Studies on herbaceous plant species in the graveyard areas of Rajshahi City. Plant Environ. Dev. 2007; 1(1): 57-60.

[12] Rahman MO and Hassan MA. Angiospermic flora of Bhawal National Park, Gazipur (Bangladesh). Bangladesh J. Plant Taxon. 1995; 2(1\&2): 47-79.

[13] Rahman MO, Uddin MZ, Tutul E, Begum M and Hassan MA. Additions to the angiospermic flora of Runctia Sal forest, Bangladesh. Bangladesh J. Plant Taxon. 2010; 17(2): 171-185.

[14] Rahman MO, Antara MT, Begum M and Hassan MA. Floristic diversity of Dhamrai Upazilla of Dhaka with emphasis on medicinal plants. Bangladesh J. Bot. 2012; 41(1): 71-85.

[15] Rahman, A H M M, Islam AKMR and Hossain M M. Taxonomy of Cucurbitaceae: Taxonomic investigation of wild \& cultivated cucurbits of Northern Parts of Bangladesh. LAP Lambert publishing, Germany, 2013; pp.1-176.

[16] Rahman A H M M, Islam A K M R and Rahman M M. The Family Asteraceae of Rajshahi Division, Bangladesh, VDM Verlag Dr. Muller Publishing House Ltd., Germany. 2011.

[17] Rahman AHMM, Islam AKRM, Zaman ATMN, Hossain MD and Afza R. Studies on the Aquatic Angiosperms of the Rajshahi University Campus. Res. J. Agri. Biol. Sci. 2007; 3(5): 474-480.

[18] Tutul E, Uddin MZ, Rahman MO and, Hassan MA. Angiospermic flora of Runctia Sal forest, Bangladesh. I. Liliopsida (Monocots) Bangladesh J. Plant Taxon. 2009;6(1): 83-90.

[19] Tutul E, Uddin MZ, Rahman MO and Hassan MA. Angiospermic flora of Runctia Sal forest, Bangladesh. II. Magnoliopsida (Dicots) Bangladesh J. Plant Taxon. 2010; 17(1): 33-53.

[20] Uddin SB and Rahman MA. Angiospermic flora of Himchari National Park, Cox's Bazar, Bangladesh. Bangladesh J. Plant Taxon. 1999; 6(1): 31-68.

[21] Uddin MZ and Hassan MA. Angiosperm diversity of Lawachara National Park (Bangladesh). Bangladesh J. Plant Taxon. 2010; 17(1): 9-22. 